\title{
Entwicklung und Stand der Initiative „Keine Bildung ohne Medien“!
}

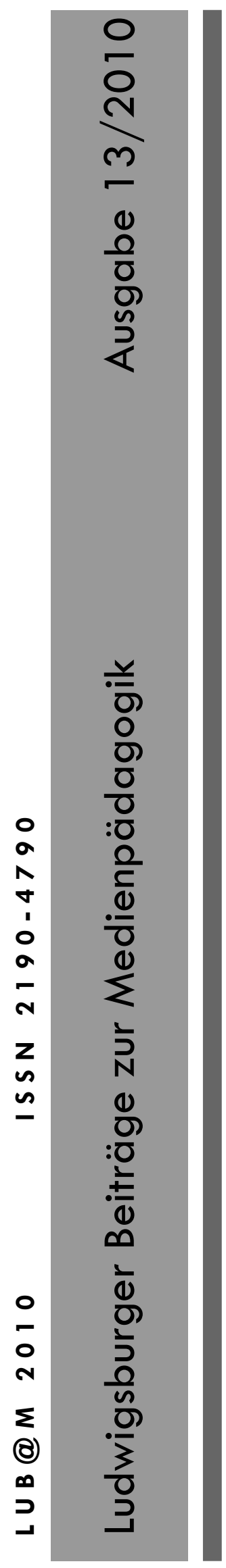

\author{
HORST NIESYTO
}

Im Frühjahr 2009 veröffentlichte die Initiative „Keine Bildung ohne Medien!“ das Medienpädagogische Manifest, das seither einen großen Zuspruch erhielt, darunter auch von bundesweiten Dachverbänden und Organisationen. Das Dokument enthält verschiedene Forderungen, um Medienpädagogik und Medienbildung in der Gesellschaft breiter zu verankern. Am 24. und 25. März 2011 wird ein bundesweiter Medienkongress „Keine Bildung ohne Medien!“ an der TU Berlin stattfinden, auf dem die Forderungen konkretisiert und mit VertreterInnen aus Bildungspolitik und Bildungsadministration diskutiert werden sollen. Von dem Kongress erhofft sich die Medienpädagogik einen wichtigen Impuls für die breite und nachhaltige Verankerung von Medienkompetenzförderung und Medienbildung in allen Bildungsbereichen. Der folgende Beitrag gibt einen Überblick zur Entstehung und zur Entwicklung der Initiative „Keine Bildung ohne Medien!“, informiert über die zentralen Anliegen und Forderungen des $\mathrm{Me}$ dienpädagogischen Manifests und bilanziert die bisherigen Aktivitäten der Initiative.

Wie kam es zu dem Medienpädagogischen Manifest und zur Initiative „Keine Bildung ohne Medien!"'?

Der Vorstand der Kommission Medienpädagogik in der Deutschen Gesellschaft für Erziehungswissenschaft (DGfE) initiierte 2007 die Diskussion über die Entwicklung einer bildungs- und professionspolitischen Initiative. Ausgangspunkt war ein Diskussionspapier, dessen Kernpunkte auf einer Mitgliederversammlung der Kommission Medienpädagogik im November 2007 in Paderborn beraten wurden. Im Mittelpunkt standen Vorschläge für professionspolitische Aktivitäten, die vor allem auf eine bessere Verankerung von Medienbildung in pädagogischen Studiengängen abzielten. Dafür sollten auch öffentlichkeitswirksame Aktivitäten mit anderen Fachgesellschaften und Einrichtungen geplant werden. Die Mitgliederversammlung unterstützte die Grundintention der Vorschläge und gab dem Kommissionsvorstand den Auftrag, Gespräche mit anderen Fachgesellschaften und Einrichtungen zu führen, um zu sondieren, ob sich eine Bereitschaft und eine inhaltliche Grundlage für gemeinsame

\section{Aktivitäten abzeichnen.}

Im Januar und Februar 2008 fanden Gespräche mit VertreterInnen der Gesellschaft für Medienpädagogik und Kommunikationskultur e.V. (GMK) und des JFF - Institut für Medienpädagogik in Forschung und Praxis statt. Es wurden verschiedene Ideen entwickelt, die von einer zu verstärkenden Netzwerkarbeit über eine gemeinsame Presseerklärung zu medienpädagogischen Handlungsbedarfen bis hin zu einer gemeinsamen professionspolitischen Tagung zum Stand der Verankerung von Medienpädagogik in neuen BA- und MA-Studiengängen reichten. Die Beratungen verdeutlichten, dass eine professionspolitische Initiative sinnvoll ist, die neben dem Hochschul- und Ausbildungsbereich alle relevanten Handlungsfelder der Medienpädagogik einbezieht. Im Laufe des Jahres 2008 entstand dann die Idee zu einem Medienpädagogischen Manifest. Dieses Manifest sollte in möglichst prägnanter Form wesentliche medienpädagogische Aufgaben und Ziele skizzieren, die dringendsten Handlungsbedarfe in verschiedenen Praxis- und Handlungsfeldern benennen und konkrete Forderungen insbesondere in Richtung Bildungspolitik und Bildungsadministration formulieren.

Obgleich es in der Vergangenheit zwar immer wieder Kontakte und Kooperationen zwischen medienpädagogischen Einrichtungen und Organisationen gab und insbesondere in der GMK zahlreiche KollegInnen und Einrichtungen Mitglied sind, so fehlte es doch an einer übergreifenden Initiative, um gemeinsam bildungs- und professionspolitische Vorstellungen in der Öffentlichkeit zu artikulieren. Es ist erfreulich und sehr positiv zu bewerten, dass es im Laufe des Jahres 2008 gelang, sich auf eine gemeinsame Einschätzung der Situation und zentraler Anliegen und Forderungen zu verständigen. Das $M e-$ dienpädagogische Manifest ist ein wichtiger Meilenstein auf dem Weg zu einem breiten gesellschaftlichen Bündnis für Medienkompetenzförderung und Medienbildung in Deutschland. $\mathrm{Zu}$ den ErstunterzeichnerInnen des Medienpädagogischen Manifests gehören der Vorstand der Kommission Medienpädagogik in der Deutschen Gesellschaft für Erziehungswissenschaft (DGfE), die Fachgruppe Medienpädagogik in der Deutschen Gesellschaft für Publizistik- und Kommunikationswissen- 
schaft (DGPuK), der Vorstand der Gesellschaft für Medienpädagogik und Kommunikationskultur e.V. (GMK), der Vorstand des JFF - Jugend, Film, Fernsehen e.V. (München) und das Hans-Bredow-Institut für Medienforschung (Hamburg).

\section{Was sind die zentralen Anliegen und Forderungen des Manifests?}

Ausgangspunkt ist die Einschätzung, dass in den letzten 20 bis 30 Jahren zwar diverse medienpädagogische Aktivitäten, Projekte und Modelle entstanden, eine breitenwirksame und nachbaltige Verankerung der Medienpädagogik aber noch nicht erreicht ist:

„Die Medienpädagogik hat in den beiden vergangenen Jahrzehnten beachtliche Fortschritte in Theorie, Forschung und Praxis erzielt. So konnte eine Reihe notwendiger, aber längst nicht hinreichender medienpädagogischer Fundamente geschaffen werden: Theoretische und empirische Arbeiten beleuchten die vielfältigen Dimensionen des Medienhandelns und die Bedeutung der Medien für Sozialisation und kulturelle Alltagspraktiken. Es gibt eine Fülle an hervorragenden medienpädagogischen Materialien für die Praxis, eine Vielzahl an überzeugenden Modellversuchen und eindrucksvollen Leuchtturmprojekten - aber es fehlt an der erforderlichen Nachhaltigkeit. Es mangelt nach wie vor an der Infrastruktur und an den organisatorischen Rahmenbedingungen in den Bildungseinrichtungen sowie an der medienpädagogischen Qualifikation der pädagogischen Fachkräfte“ (Manifest).

Deshalb fordert das Medienpädagogische Manifest eine umfassende Förderung der Medienpädagogik in Wissenschaft und Forschung sowie auf allen Ebenen der Erziehungs- und Bildungspraxis. Notwendig sind nicht nur programmatische Überlegungen sowie eine auf Jahre angelegte strategische Planung, sondern insbesondere auch personelle, infrastrukturelle und finanzielle Investitionen auf Länder- und Bundesebene. Dabei gibt es keinen zentralen Ort für die Medienkompetenzförderung, auf den die Kräfte zu konzentrieren sind - es sind alle Erziehungs- und Bildungsbereiche und deren Institutionen zu berücksichtigen, auch die außerschulische Kinder- und Jugendarbeit, die berufliche Aus- und Fortbildung, die Erwachsenen-, Familien- und Seniorenbildung sowie das Internet mit seinen social communities als neue Orte für Information, Kommunikation und Bildung.

Die zentrale Aufgabe - so das Medienpädagogische Manifest - besteht heute darin, die Medienpädagogik von einer Phase der Modellprojekte und einzelnen Aktionen auf lokaler und regionaler Ebene zu einer Phase struktureller Veränderungen weiter zu entwickeln. Hierfür reichen punktuelle Maßnahmen und diverse
Informations- und Beratungsangebote im Internet und in anderen Medien längst nicht mehr aus. Kinder und Jugendliche nutzen zwar sehr intensiv Medien, aber es mangelt in Kindergärten, Schulen und außerschulischen Einrichtungen - in der Breite gesehen - an Angeboten, die zu einem kreativen und kritischen Umgang mit Medien anregen. Viele Eltern und Erziehende in allen pädagogischen Bereichen sind auf dem Hintergrund der umfassenden Mediatisierung der Lebenswelten von Kindern und Jugendlichen hinsichtlich ihrer medienerzieherischen Verantwortung unsicher. In dieser Situation ist es geboten, Medienpädagogik dauerhaft in allen Bildungbereichen zu verankern.

Das Medienpädagogische Manifest benennt Aufgaben, die vordringlich sind und stellt hierzu Forderungen auf:

- Damit alle Kinder und Jugendlichen die Chance erhalten, ihre Medienkompetenzen $\mathrm{zu}$ erweitern, müssen medienpädagogische Programme vor allem in den Einrichtungen der Elementarpädagogik sowie in der Jugend-, Familien- und Elternbildung verstärkt werden.

- In der aktuellen Diskussion zur Schulreform (z.B. Ganztagsschulen) müssen für alle Schulformen auch Bildungsstandards für $\mathrm{Me}$ dienkompetenz. vereinbart und entsprechende medienpädagogische Inhalte in Curricula verbindlich verankert werden. Dieser Prozess muss durch Evaluationsstudien und Programme zur Qualitätssicherung sowie durch nachhaltige Fortbildungsmaßnahmen für alle Lehrpersonen und pädagogischen Fachkräfte unterstützt werden.

- Einen besonderen Schwerpunkt stellen pädagogische Angebote für Heranwachsende aus Migrationskontexten und bildungsbenachteiligten Milieus sowie Angebote zur geschlechtersensiblen Arbeit dar. Dafür müssen stärker als bisher die Einrichtungen der außerschulischen Kinder- und Jugendarbeit genutzt werden. Eine Intensivierung der Medienprojekte in diesem Bereich ist durch die Verbesserung der Infrastruktur und der personellen Ausstattung sowie durch kontinuierliche öffentliche Mittel zu sichern. Medienpädagogik ist im Kontext kultureller Bildung erheblich mehr zu fördern.

- In der Ausbildung von Erzieher/innen, Lehrer/innen, Erwachsenenbildnern/innen und Sozialpädagogen/innen ist generell eine medienpädagogische Grundbildung als verbindlicher Bestandteil der pädagogischen Ausbildung zu verankern. Daneben müssen spezifische medienpädagogische Ausbildungen in Form von Master-Studiengängen und als Wahlpflichtbereiche in anderen Studiengängen angeboten werden. Voraussetzung hierfür ist der erhebliche Ausbau medienpädagogischer 
Professuren und Lehrstühle mit Infrastruktur an den Hochschulen.

- Im Bereich der medienpädagogischen Forschung bedarf es erheblich mehr Mittel und Förderprogramme insbesondere für den Bereich der Mediensozialisationsforschung und der medienpädagogischen Begleit- und Praxisforschung.

Das Medienpädagogische Manifest wurde anlässlich einer Fachtagung über Computerspiele an der Universität Magdeburg im März 2009 veröffentlicht. In den folgenden Monaten stand eine Unterschriftensammlung zu den Anliegen und Forderungen des Manifests im Mittelpunkt. Am Rande des GMK-Forums 2009 beschloss die Initiative, die Unterschriftenaktion auf elektronischer Basis fortzuführen und zugleich Vorbereitungen für die Durchführung eines bundesweiten Medienkongresses zu treffen. Es gelang noch im Dezember 2009 eine Website aufzubauen, die Pressearbeit aufzunehmen und die elektronische Unterschriftenaktion zu starten.

Bisherige Resonanz auf die Initiative „Keine Bildung ohne Medien!“ im Kontext weiterer Initiativen zur Förderung von Medienkompetenz und Medienbildung in Deutschland

Bislang unterzeichneten über 1000 Personen und Einrichtungen das Medienpädagogische Manifest, darunter mehrere Dachverbände und Initiativen auf Bundesebene, auch einzelne Bildungsund MedienpolitikerInnen aus verschiedenen Parteien. Organisationen wie z. B. „Schulen ans Netz", die Initiative D21, der Deutsche Bibliotheksverband, Landesjugendringe u. a. Organisationen informierten ausführlich über die Intentionen und Forderungen des Medienpädagogischen Manifests und die Aktivitäten der Initiative. Während sich Tageszeitungen mit der Berichterstattung eher zurückhielten, gab es im Onlinebereich diverse Hinweise und Berichte, u. a. im Rahmen eines Interviews mit ARD.de. Die an der Initiative beteiligten Partnerorganisationen verdeutlichten in verschiedenen lokalen und überregionalen Veranstaltungen die Anliegen des Manifests. In der Sektion Medienpädagogik der DGfE engagieren sich seit dem Frühjahr 2010 mehrere KollegInnen und gründeten Arbeitsgruppen, um die Forderung nach einer medienpädagogischen Grundbildung in verschiedenen Ausbildungsbereichen zu konkretisieren. Die Initiative wurde für Vorträge, Stellungnahmen und Teilnahme an Podiumsdiskussionen und Workshops angefragt, z. B. im Rahmen der GMW-Jahrestagung „Lernen im digitalen Zeitalter“ in Berlin, der 33. Stuttgarter Tage der Medienpädagogik, des D21-Jahreskongresses in Hannover, einer Fachkonferenz des Bundesbildungsministeriums im Juli 2010, den $\mathrm{MaC}^{*}$ days 2010 in Josefstal oder der Auftaktveranstaltung des Bundesjugendministeriums
„Dialog Internet“ im Herbst 2010. Die Landesanstalt für Kommunikation BadenWürttemberg (LFK) erklärte sich bereit, den geplanten Medienkongress in Berlin finanziell zu fördern. Dadurch wurde es u. a. möglich, ein Projektbüro der Initiative an der PH Ludwigsburg einzurichten, um den Kongress auf solider Basis vorbereiten und durchführen zu können. Auch die Landesanstalt für Medien in Nordrhein-Westfalen (LfM) in Düsseldorf wird den Kongress fördern. Beide Landesanstalten engagieren sich seit vielen Jahren regional und auch überregional bei der Förderung von innovativen medienpädagogischer Projekten und in der medienpädagogischen Forschung.

Die zahlreichen Kontakte, Aktivitäten und Gespräche verdeutlichen: Das Medienpädagogische Manifest kam zum richtigen Zeitpunkt. In der Öffentlichkeit wächst das Bewusstsein, dass in einer mediatisierten Gesellschaft die breite und nachhaltige Förderung von Medienkompetenz und Medienbildung zu den unverzichtbaren Bildungsaufgaben gehört. So engagieren sich auch außerhalb des Kernbereichs der Medienpädagogik viele Fachkräfte in Initiativen und Organisationen, wie z. B. D21, educamp, GMW oder re-publica, die sich mit der Entwicklung digitaler Medien und ihrer Bedeutung für Bildung, Politik, Wissenschaft und Wirtschaft befassen. Hierzu gehören auch öffentlich-rechtliche Rundfunk- und Fernsehsender, neue Unternehmen im Bereich der Medien- und Kommunikationswirtschaft mit Online-Portalen für Kinder und Jugendliche oder Buch-, Schulbuch-, Zeitungs- und Zeitschriftenverlage. In den letzten Jahren entstanden auch Unternehmen insbesondere im Bereich ComputerLernsoftware, die pädagogisch relevante Dienstleistungen und Produkte offerieren. Auch die Verbraucherbildung unternimmt in letzter Zeit vermehrt Anstrengungen, den Bereich Medienkompetenz in ihr Beratungsprofil zu integrieren.

Gleichzeitig wird deutlich, dass nach wie vor eine Kluft zwischen allgemeinen bildungspolitischen Proklamationen einerseits und tatsächlichen breitenwirksamen Maßnahmen zur Förderung von Medienkompetenz und Medienbildung besteht. Zwar entstanden in verschiedenen Bundesländern Initiativen zur besseren Vernetzung vorhandener medienpädagogischer Angebote. Die „Länderkonferenz Medienbildung“ legte Positionspapiere vor, um auf eine bessere und nachhaltige Verankerung von Medienbildung in Schule und Unterricht hinzuwirken. Die „Gemischte Kommission“ der Kultusministerkonferenz beabsichtigt eine Aktualisierung der Erklärung „Medienpädagogik in der Schule“ aus dem Jahre 1995. Das BMBF setzte eine Expertenkommission ein, die 2009 den Bericht „Kompetenzen in einer digital geprägten Kultur" erarbeitete. Das BMBF und das BMFSFJ luden zu oben genannten Fachkonferenzen ein, um Fragen der Medienkompetenz- 
förderung und der Medienbildung zu erörtern. Im Deutschen Bundestag konstituierte sich 2010 eine Enquetekommission „Internet und digitale Gesellschaft" und bildete eine spezielle Projektgruppe zum Thema „Medienkompetenz".

Obgleich diese u. a. Entwicklungen zeigen, das in letzter Zeit deutlich mehr Bewegung in die öffentliche Diskussion über die Notwendigkeit einer breiten Medienkompetenzförderung gekommen ist, so zeichnen sich bislang keine konkreten Maßnahmen zu einer breitenwirksamen und nachhaltigen Verankerung von Medienbildung und Medienkompetenzförderung $\mathrm{ab}$, die über die bisherigen Fördermaßnahmen deutlich hinausgehen. Zwar gibt es mehr Raum für Diskussion und staatliche Akteure sind bestrebt, vorhandene Angebote besser sichtbar zu machen und zu vernetzen. Medienbildung hat aber immer noch keine Priorität in den bildungspolitischen Beschlüssen. So kündigte die CDU/CSU/FDPBundesregierung in ihrer Koalitionsvereinbarung 2009 an, dass sie die Lehrerausbildung an deutschen Hochschulen stärken möchte: „Der Erhöhung der Medienkompetenz kommt dabei eine besondere Rolle zu“. Auf entsprechende Maßnahmen wird seither vergeblich gewartet; offensichtlich sind ursprünglich geplante Maßnahmen in Zusammenhang mit dem aktuellen „Sparhaushalt“ gestrichen worden. Die (bildungs-)politischen Prioritäten werden nach wie vor anders gesetzt ...

Mit Blick auf die Situation in verschiedenen Bundesländern und Handlungsfeldern ist diese Einschätzung sicherlich zu differenzieren. Hier gibt es teilweise Bestrebungen, die stärker in Richtung Nachhaltigkeit gehen und darauf hoffen lassen, dass sich in den nächsten Jahren mehr bewegt. Aktuell zu nennen sind u. a. das „10-Punkte-Programm“ der Landesregierung in Rheinland-Pfalz sowie die Ankündigung in der Koalitionserklärung der neuen Landesregierung in Nordrhein-Westfalen, NRW zum „Medienkompetenzland Nummer 1" in Deutschland zu machen. Wenn es hier zu einem produktiven Wettbewerb um die besten Konzepte und wirkungsvolle Fördermaßnahmen zwischen den Bundesländern kommt, ist dies zu begrüßen. Die Initiative „Keine Bildung ohne Medien!“ geht davon aus, dass neben bundesweiten Rahmenbedingungen vor allem auf regionaler und lokaler Ebene entscheidende Fortschritte zu erzielen sind. Um die aktuelle Situation der Medienkompetenzförderung zu dokumentieren und Raum für das Öffentlichmachen von Analysen, Positionspapieren und Statements zu geben, wurde auf der Website der Initiative eine Bundesländer-Karte eingerichtet, die sukzessive ergänzt wird.

\section{Anmerkungen zu einzelnen konzep- tionellen Fragen, insbesondere im Bereich Medien und Lehrerbildung}

Erfahrungen aus den bisherigen Aktivitäten und Gesprächen zeigen, dass es bei konzeptionellen Vorstellungen zur Medienkompetenzförderung mitunter erhebliche Unterschiede gibt. Zwar ist den meisten AkteurInnen - auch im Bereich der allgemeinen und der beruflichen Bildung - bewusst, dass eine Reduktion auf eine informationstechnische Bildung nicht ausreicht. Der Bericht der Expertenkommission des BMBF aus dem Jahre 2009 benannte hier klar die Mehrdimensionalität von Medienbildung für die Persönlichkeitsentwicklung, für die gesellschaftliche Teilhabe und für die Entwicklung von Ausbildungs- und Erwerbsfähigkeit. Allerdings gibt es nach wie vor starke Tendenzen, Medienkompetenzförderung und Medienbildung auf die instrumentelle Kenntnis bestimmter Bedienkompetenzen und Verhaltensweisen im Internet zu reduzieren, verbunden mit einem relativ starren Verständnis des Erreichens von Kompetenzniveaus in der schulischen Medienbildung. Hier ist es wichtig, soziale, ästhetische und kulturelle Dimensionen mehr ins Blickfeld $\mathrm{zu}$ rücken und den Prozesscharakter von Medienbildung zu verdeutlichen.

Ein anderer Punkt betrifft die Frage, inwieweit Maßnahmen zur Medienkompetenzförderung angesichts der neuen Generation von „Digital Natives" zu relativieren sind. Auf dem D21Jahreskongress 2010 in Hannover empfahl z. B. Bundesinnenminister Dr. Thomas de Maizière „mehr Gelassenheit“ bei Maßnahmen zur Medienkompetenzförderung, da doch sehr viele junge Menschen recht kompetent Medien nutzen. Er sieht weniger im Bereich Bildung und Ausbildung, sondern mehr im Bereich der Medientechnologie Förderbedarf, z. B. mehr Breitbandverkabelung insbesondere für ländliche Regionen. In der Podiumsrunde unterstützte ihn Constanze Kurz, Sprecherin des „Chaos Computer Clubs“. Ihr Argument: Die jungen Lehrkräfte von heute kommen aus einer Generation, die mit Medien aufgewachsen ist und die deshalb eine bessere Medienkompetenz für den Lehrerberuf mitbringe.

Zweifelsohne sind viele ländliche Regionen nach wie vor bei der Breitbandverkabelung benachteiligt, und es ist auch richtig, dass vermehrt Jahrgänge in die Hochschulausbildung und dann in die Schulen kommen, die mit dem Internet und digitalen Medien aufgewachsen sind. Es gibt auch seit ein paar Jahren Initiativen wie educamp, in der sich viele junge WissenschaftlerInnen und Lehrkräfte engagieren, um Social Media, Web 2.0 und innovative Lehr-/Lernmethoden mit digitalen Medien in Bildungs- und Lernprozesse zu integrieren. Gleichzeitig deuten Studien und Beobachtungen unter Lehramtsstudierenden und in Schulen darauf hin, dass es nach wie vor 
eine Kluft zwischen formellen (schulischen) und informellen Lernprozessen mit Medien gibt (zsf. Herzig/ Grafe 2010). Offensichtlich ist es noch nicht gelungen, digitale Medien als Brücke zur Verbindung von formellen und informellen Bildungs- und Lernprozessen hinreichend zu nutzen und entsprechende Potenziale nachhaltig in institutionalisierte Prozesse zu integrieren. In der Breite gesehen ist Medienbildung an sehr vielen Schulen noch nicht angekommen (Tulodziecki 2006).

Es ist offensichtlich kurzschlüssig anzunehmen, die neue „Netzgeneration“ werde quasi im Selbstlauf Medienbildung an Schulen fest verankern. Die Netzgeneration ist im Plural zu denken: es gibt unterschiedliche Medienumgangsweisen. Weitere Studien sind notwendig, um mögliche Veränderungsprozesse in den medialen Habitusformen von Lehramtstudierenden und Lehrkräften analysieren zu können. Hierzu gehört auch die Frage, inwieweit Bildungsangebote in der 1. Phase der Lehrerbildung zu einer Veränderung von medialen Habitusformen beitragen können und was aus diesen Impulsen in der 2. Phase und im Lehreralltag an Schulen wird. Auch geht es um grundlegende Fragen der Funktion und des Selbstverständnisses von Schule heute und der Bedeutung von Medienbildung für eine Schulentwicklung, um Potenziale für schülerorientierte und selbstorganisierte Bildungsund Lernprozesse erheblich intensiver zu nutzen. Grundlagen hierfür wie z. B. die medienbiografische Reflexion der eigenen Medienerfahrungen, Medienwissen zu unterschiedlichen Aspekten und Medienbildung als wesentlicher Teil von Schulentwicklung könnten im Rahmen einer medienpädagogischen Grundbildung angeeignet werden.

Es reicht nicht aus, dass sich Lehrkräfte auf Moderationsaufgaben konzentrieren - dies wird in Verbindung mit dem Hinweis auf die Generation der „Digital Natives“ oft empfohlen. Um Bildungs- und Erziehungsaufgaben in der mediatisierten Welt wahrnehmen zu können, müssen Lehrkräfte über grundlegende Medienkompetenzen und medienpädagogische Kompetenzen verfügen. Hierzu gehören u. a. medienästhetische, -technische, -ethische, -didaktische Kenntnisse und Fähigkeiten. Wenn Lehrkräfte einen differenzierten Umgang mit Medien fördern und auf die Fragen von SchülerInnen überzeugend und glaubwürdig eingehen möchten, benötigen sie selbst ein Medienwissen und eine reflektierte Haltung im Umgang mit Medien. Auf dieser Grundlage können sie Bildungsprozesse im Sinne von Selbstorganisationskompetenzen bei SchülerInnen mit auf den Weg bringen, gerade was berufsbezogenes Wissen und Reflexionswissen über die Mediengesellschaft und die Risiken im Umgang mit digitalen Technologien betrifft (vgl. Moser 2010, S. 76). Es geht nicht um ein „Beibringen“, sondern um eine
„Ermöglichungsdidaktik“" (Moser, ebd.), zu der auch das informelle Lernen mit und über Medien bis hin zu Konzepten der Peer-Education gehören, z. B. im Rahmen von „SchülerMedienmentoren-Programmen“.

SchülerInnen erwarten hier einiges von ihren LehrerInnen! Dies konnte u. a. eine OnlineUmfrage zum Thema „Medien und Schule"belegen, die die Initiative „Keine Bildung ohne Medien!““ im Sommer 2010 auf der Plattform schülerVZ durchführte. Die Ergebnisse der Befragung, an der sich über 6000 Jugendliche und junge Erwachsene im Alter von 12 - 19 Jahren beteiligten, sind deutlich. Die Jugendlichen wünschen sich eine Schule, in der Medien ein selbstverständlicher Bestandteil sind. Nicht nur, weil Schule mit Medien mehr Spaß macht, sondern weil Medien zu ihrem Alltag gehören und sie sich eine berufliche Zukunft ohne umfassende Medienkompetenz kaum mehr vorstellen können. Die Befragten sprechen sich für eine starke Verankerung von Medien in der Schule aus: 90 $\%$ sind dafür, dass Medien im Unterricht eine größere Rolle spielen, $77 \%$ in Projekttagen und $56 \%$ in Nachmittags-AG's. Sichtbar wird auch ihr Bedarf an Orientierung und Reflexion. Wie geht man ,richtig' mit Medien um? Welche Risiken und Gefahren gibt es, z.B. beim Umgang mit privaten Daten oder beim Thema CyberMobbing? Von ihren Lehrerinnen und Lehrern erwarten die Jugendlichen Handlungsempfehlungen und konkrete Unterstützung. $\mathrm{Ob}$ die Lehrpersonen auf diese Aufgabe vorbereitet sind, ist aus Sicht vieler Schülerinnen und Schüler fraglich: $56 \%$ von ihnen sind der Ansicht, dass ihre Lehrerinnen und Lehrer wenig oder nichts von ihrem Medienalltag wissen. Gefragt nach den Gründen geben $86 \%$ an, dass Lehrkräften eigene Erfahrungen mit (neuen) Medien fehlen, $74 \%$ sind der Meinung, dass es ihnen an Interesse für Medien mangelt.

Aus anderen Studien ist bekannt, dass insbesondere Kinder und Jugendliche aus bildungsbenachteiligten Sozialmilieus über deutlich weniger Anregungspotenziale in ihren Sozialisationskontexten verfügen (u. a. Wagner 2008). Hier kommt dem Sozialisationsfeld Schule eine wichtige Aufgabe zu, um insbesondere durch anschauliche und praxisbezogene Formen der Medienbildung reflexive Bildungsprozesse $\mathrm{zu}$ fördern (siehe hierzu die in Modellprojekten erprobten Erfahrungen, z. B. bei Kutscher u. a. 2009 und Niesyto u. a. 2007). Voraussetzung hierfür ist die Bereitschaft und Fähigkeit von Lehrkräften, zielgruppenspezifische medienpädagogische Konzepte für den Unterricht zu entwickeln. Lehrkräfte, die Medienerfahrungen von Kindern und Jugendlichen aus bildungsbenachteiligten Milieus abwerten und eine oberflächlichkritische Haltung gegenüber Medien haben, sind nicht in der Lage, vorhandene Potenziale bei SchülerInnen $\mathrm{zu}$ fördern und tragen zur 
Reproduktion von sozialer Benachteiligung und Ungleichheit bei.

In Gesprächen über die Etablierung einer verbindlichen Mediengrundbildung an Hochschulen kommt mitunter der Hinweis, dass die Wahlfreiheit der Studierenden dadurch eingeschränkt werde. Medienkompetenz sei nur ein Thema unter vielen. Gesellschaftlich relevante Themen wie Gender, Migration, Umgang mit Heterogenität, Umweltbildung oder Gesundheitserziehung müssten genauso berücksichtigt werden. Es sei nicht möglich, einzelne dieser Aufgaben herauszugreifen und einseitig in der Lehrerbildung in den Vordergrund zu rücken.

Es ist wichtig, angehende pädagogische Fachkräfte dadurch für Medienbildung zu sensibilisieren und zu gewinnen, indem sie die Bedeutung, die Potenziale und den Mehrwert von Medienbildung für Bildungs- und Lernprozesse selbst erfahren. Das beginnt mit eigenen medienbiografischen Reflexionen, dem Kennenlernen der Medienwelten von Kindern und Jugendlichen, Überlegungen zum Transfer von alltäglichen Medienpraxen in formelle Lernsituationen, niedrigschwelligen Möglichkeiten zu eigenen Medienproduktionen in Verbindung mit unterschiedlichen Themen und Fragestellungen. Neben projektbezogenen Seminaren und Werkstätten sind hochschuldidaktisch kreative und innovative Lösungen gefragt, gerade in Zusammenhang mit orientierenden, größeren Vorlesungen.

Die wichtige Bedeutung einer motivationsfördernden Seminar- und Vorlesungspraxis erübrigt allerdings nicht die Frage danach, was neben Wahl- und Vertiefungsangeboten - zu einem zeitgemäßen Fundamentum der Lehrerbildung gehört. Hier bedarf es pädagogischprofessioneller Vorgaben und auch bildungspolitischer Rahmenbedingungen. Diese Vorgaben und Rahmenbedingungen sind nicht unveränderlich, sondern Bestandteil der Professionsentwicklung und des gesellschaftlichen Wandels. Wer heute die Einschätzung teilt, dass wir in einer Mediengesellschaft leben und nahezu alle Bereiche unseres Lebens und unserer Welterfahrung medienvermittelt sind, der kann sich auf Dauer nicht der Einschätzung entziehen, dass Medienbildung im Sinne von Persönlichkeitsbildung und Gesellschaftsfähigkeit ein integraler und fest verankerter Bestandteil von schulischer Bildung sein muss . Die 1. Phase der Lehrerbildung hat hierfür bei allen Studierenden ein Fundamentum zu legen. Das Lehramtsstudium sollte allerdings so gestaltet werden, dass Studierende nicht durch Stofffülle und kleinmaschige Prüfungsbedingungen in ein Korsett gezwängt werden, welches die Offenheit für eigenständige Erkenntnis- und Lernprozesse erstickt. Gerade die Medienbildung sollte einen Beitrag leisten, um die Dominanz instrumenteller Denkmuster (z.B. Fixierung auf reproduzierbares Wissen und ,Abhaken' von Scheinen) zu hinterfragen und durch die Förderung selbstorganisierter Lernprozesse zu überwinden. Hierzu bedarf es aber nicht nur geeigneter hochschuldidaktischer Konzepte, sondern auch entsprechender personeller und infrastruktureller Ressourcen. Deshalb ist die Forderung, dass an allen Hochschulen mit pädagogischen Studiengängen Professuren mit einer klaren Denomination im Bereich Medienpädagogik und entsprechende akademische Mitarbeiterstellen einzurichten sind; hierzu gehört auch eine bedarfsgerechte und funktionierende mediale Infrastruktur.

Fakt ist, dass gerade jene Themen- und Kompetenzfelder, die nicht klar einem Schulfach zugeordnet werden können, es schwer haben, sich in der schulischen Bildung zu behaupten bzw. überhaupt einen adäquaten Platz zu erhalten. Die Situation in den Bildungsplänen der einzelnen Bundesländer stellt sich durchaus unterschiedlich dar. Insgesamt wird aber deutlich, dass die sog. Querschnittskompetenzen - zu denen auch Medienkompetenz zählt - nicht entsprechend ihrem gesellschaftlichen Stellenwert und in ihrer Bedeutung für die Persönlichkeitsbildung junger Menschen in der schulischen Bildung verankert sind. Hier stellt sich zentral die Frage, welche Schulen wir heute brauchen, wie Bildungs- und Erziehungsaufgaben gewichtet werden und wie die sog. Querschnittskompetenzen ein größeres Gewicht bekommen können. Für eine Schule, die zukunftsfähig bleiben möchte, wird kein Weg daran vorbeigehen, mehr Raum für die Auseinandersetzung mit zentralen Lebensthemen der SchülerInnen zu schaffen. Es geht also nicht darum, einzelne Themenfelder und Kompetenzbereiche gegeneinander auszuspielen, sondern nach Wegen zu suchen, wie insgesamt Schlüsselthemen nachhaltig in der schulischen Bildung verankert werden können. Eine einseitige Orientierung am Fächerprinzip ist dafür nicht geeignet.

Beispiel Medienbildung: Hier ist die Situation, dass in den Fächern oft die Zeit fehlt, um auch spezifische Fragen der Medienbildung und Medienerziehung zu thematisieren. Weder der nur fächerintegrative Ansatz noch die Forderung nach einem gesonderten, neuen Schulfach „Medienbildung" erscheinen sinnvoll. Erfolgversprechend sind curriculare Konzepte, die im Zusammenspiel von Fächern und fächerübergreifenden Themenfeldern Raum für mediale Bildungsprozesse eröffnen. Hierzu gehören auch „Fenster“ in bestehenden Fächern, in denen mediales Wissen gezielt vermittelt und zum Bestandteil von Evaluation und Selbstevaluation wird (Moser 2010, S. 73).

Die Postulate vom selbstregulierten, selbstbestimmten und mobilen Lernen können erst dann realisiert werden, wenn LehrerInnen einen schü- 
lerorientierten Unterricht machen, mehr Projektarbeit, freie und offene Unterrichtsformen fördern - und hierin in vielfältiger Weise Bildung und Lernen mit und über Medien integrieren. Hierfür sind nicht nur die Klassengrößen zu reduzieren und mehr Kooperationen mit außerschulischen Lernorten - insbesondere im Kontext von Ganztagesangeboten - zu entwickeln. Es bedarf auch einer kritischen Überprüfung der Stofffülle in einzelnen Fächern (in Richtung Mindeststandards für alle und wahlbezogenen Vertiefungsmöglichkeiten). Struktur und Angebote in der 1. Phase der Lehrerbildung sollten hierauf vorbereiten. Dies setzt voraus, den interdisziplinären Themenfeldern und Querschnittskompetenzen erheblich mehr Gewicht beizumessen und an den einzelnen Hochschulstandorten nach Lösungen zu suchen, wie sowohl eine (domänenspezifische) Grundbildung in diesen Themenfeldern gewährleistet als auch Verknüpfungsmöglichkeiten zwischen den einzelnen interdisziplinären Themenfeldern sowie mit den Fächern realisiert werden können. Auch sollte bei anstehenden Neustrukturierungen der Lehrerbildung und der Forschreibung von Rahmenrichtlinien zur Medienpädagogik und Medienkompetenzförderung darauf geachtet werden, dass Konzepte zwischen den verschiedenen Phasen der Lehrerbildung erheblich besser untereinander abgestimmt werden.

Schließlich: Es wäre eine verkürzte Analyse, allein in der unzureichenden medienpädagogischen Grundbildung die Ursache für die fehlende nachhaltige und breite Verankerung von Medienbildung an Schulen zu suchen. Festzustellen sind auch Defizite im Bereich der Schulentwicklung, der entsprechenden Qualifizierung des Leitungspersonals, keine hinreichende Verankerung von Mindeststandards der Medienkompetenzbildung in bundesweiten Bildungsstandards und - damit verknüpft - eine unzureichende Evaluation und Qualitätssicherung im Bereich der schulischen Medienbildung in den Bundesländern. Deshalb ist es notwendig, auf verschiedenen Ebenen anzusetzen. Dennoch kommt der medienpädagogischen Grundbildung in der 1. Phase der Lehrerbildung eine Schlüsselfunktion zu. Kammerl/ Ostermann (2010) sprechen in ihrer Situationsanalyse zur Medienkompetenzförderung in Schulen von einem "Teufelskreis“ fehlender Medienbildung:

„Medienbildung wird in vielen Schulen noch zu wenig berücksichtigt. Die Studienanfänger, die sich für ein Lehramtsstudium entscheiden, bringen wenige Kompetenzen darin mit und die meisten von ihnen erfahren keine oder kaum Ausbildung in diesem Bereich (...) Derart unvorbereitete und nicht besonders medienaffine Studienabsolventen stoßen im Referendariat auf eine Schulpraxis, in der Medienbildung nur gering in den Fachunterricht integriert ist und eher als additive Aufgabe betrachtet wird, deren
Umsetzung derzeit noch kaum oder gar nicht überprüft wird (...) Dieser ,Teufelskreis’ muss durchbrochen werden“ (ebd., S. 49).

Was in der 1. Phase der Lehrerbildung nicht grundgelegt wird, schleppt sich als Defizit in den weiteren Phasen fort.

\section{Ausblick}

Die im vorigen Kapitel diskutierten Punkte, die vor allem den Bereich Medien und Lehrerbildung betreffen, ließen sich durch weitere Punkte aus anderen Praxis- und Handlungsfeldern ergänzen. Der Kongress „Keine Bildung ohne Medien!“" wird am 24./ 25. März 2011 in Berlin Gelegenheit zu Diskussion und Beratung bieten. Der Kongress hat folgende Zielsetzungen:

- Sensibilisierung der Öffentlichkeit für die Notwendigkeit einer breiten Förderung von Medienkompetenz in verschiedenen Handlungsfeldern.

- Diskussion und Konkretisierung der Vorschläge und Forderungen des Medienpädagogischen Manifests mit allen interessierten Kräften aus unterschiedlichen gesellschaftlichen Bereichen.

- Dialog mit Verantwortlichen in Bildungspolitik und Bildungsadministration über die zentralen Aufgaben und die zu ergreifenden Maßnahmen.

- Verständigung über die zentralen Aufgaben und Maßnahmen, um Medienkompetenzförderung nachhaltig in allen Bildungsbereichen zu verankern.

Der Kongress wendet sich an Medienpädagogen/innen, pädagogische Fachkräfte in unterschiedlichen Handlungsfeldern, Vertreter/innen aus Politik, Bildungsadministration, kommunaler Kultur- und Bildungsarbeit, dem Bibliothekswesen, Vertreter/innen aus sozialen und kirchlichen Organisationen und Verbänden, Medieninstitutionen und -wirtschaft.

Ziel des ersten Tages ist ein intensiver Austausch über wesentliche Anliegen des Medienpädagogischen Manifests. Es gibt die Möglichkeit zur Teilnahme an 13 Arbeitsgruppen, die sich vor allem auf die thematischen Schwerpunkte des zweiten Tages beziehen: Medienkompetenzförderung in der frühkindlichen Bildung und Erziehung, in der Schule, in der außerschulischen Jugendarbeit und Jugendbildung und in sonderund integrationspädagogischen Handlungsfeldern, in der Eltern- und Familienbildung, in der Erwachsenen- und Seniorenbildung. In diesen und weiteren Arbeitsgruppen werden Fragen einer medienpädagogischen Grundbildung von pädagogischen Fachkräften, die Relevanz des Bildungsortes Internet sowie soziale, bildungsund geschlechtsbezogene Aspekte integriert, ebenso die Vernetzung zwischen den einzelnen Handlungsfeldern, z.B. im Kontext gemeinwesenbezogener Aktivitäten und Kooperationen 
zwischen schulischer und außerschulischer Medienbildung.

Am zweiten Tag steht der Dialog zwischen der Initiative „Keine Bildung ohne Medien!“ und Vertreterinnen und Vertretern aus Bildungspolitik, -administration und anderen Bereichen im Zentrum. Thematische Schwerpunkte bilden die Medienkompetenzförderung an Schulen und Hochschulen sowie die Medienkompetenzförderung in der frühkindlichen Bildung und in der außerschulischen Bildung (verschiedene Alters- und Handlungsbereiche, siehe oben). In diesen Dialog werden seitens der Initiative die zentralen Ergebnisse aus den Arbeitsgruppen am ersten Tag eingebracht. Übergreifende Fragen werden sein:

- Wo bestehen aktuell die dringendsten Handlungsbedarfe?

- Welche Faktoren stehen einer umfassenden Medienkompetenzförderung bislang entgegen?

- Wie können Medienpädagogik und Medienbildung in Wissenschaft und Forschung sowie auf allen Ebenen der Erziehungs- und Bildungspraxis realisiert und nachhaltig verankert werden?

Dank der Unterstützung von Angela Ittel, Professorin am Institut für Erziehungswissenschaft an der TU Berlin, kann der Kongress in Räumen der Technischen Universität Berlin stattfinden. Erste Informationen zum Kongressprogramm erscheinen im Dezember 2010, die Anmeldemöglichkeit beginnt im Januar 2011. Die Veranstalter - die Erstunterzeichner-Organisationen des Medienpädagogischen Manifests - freuen sich über eine aktive Beteiligung von möglichst vielen Kolleginnen und Kollegen in den Arbeitsgruppen und auf dem Kongress!

\section{Literatur}

Biermann, Ralf (2009): Der mediale Habitus von Lehramtsstudierenden. Eine quantitative Studie zum Medienhandeln angehender Lehrpersonen. Wiesbaden: VS Verlag.

Billes-Gerhart, Elke (2009): Medienkompetenz von Lehramtsstudierenden. Eine empirische Beobachtung, Analyse und Interpretation der Orientierungs- und Bewertungsschemata von angehenden Lehrkräften. Göttingen: $\mathrm{Cu}$ villier.

Henrichwark, Claudia (2009): Der bildungsbezogene mediale Habitus von Grundschulkindern. Eine empirische Studie zur Reproduktion sozialer Ungleichheit in Schule und Familie. Dissertation. Wuppertal. Online verfügbar unter http://elpub.bib.uni-wuppertal. de/servlets/DerivateServlet/Derivate
Herzig, Bardo/ Grafe, Silke (2007): Digitale Medien in der Schule. Standortbestimmung und Handlungsempfehlungen für die Zukunft; Studie zur Nutzung digitaler Medien in allgemein bildenden Schulen in Deutschland. Bonn: Deutsche Telekom.

Herzig, Bardo/ Grafe, Silke (2010): Digitale Medien in Schule und Alltagswelt. Zur Verbindung von formellen und informellen Lernprozessen. In: Bachmair, Ben (Hg.): Medienbildung in neuen Kulturräumen. Wiesbaden: VS-Verlag, S. 183-195.

Kammerl, Rudolf/ Ostermann, Sandra (2010): Medienbildung - (k)ein Unterrichtsfach? Eine Expertise zum Stellenwert der Medienkompetenzförderung in Schulen. Hamburg: Medienanstalt Hamburg/ Schleswig-Holstein.

Kommer, Sven (2010): Kompetenter Medienumgang? Eine qualitative Untersuchung zum medialen Habitus und zur Medienkompetenz von SchülerInnen und Lehramtsstudierenden. Leverkusen: Budrich UniPress Ltd.

Kutscher, Nadia/ Klein, Alexandra/ Lojewski, Johanna/ Schäfer, Miriam (2009): Medienkompetenzförderung für Kinder und Jugendliche in benachteiligten Lebenslagen. Düsseldorf: Landesanstalt für Medien Nordrhein-Westfalen (LfM).

Moser, Heinz (2010): Die Medienkompetenz und die ,neue' erziehungswissenschaftliche Kompetenzdiskussion. In: Herzig, Bardo/ Meister, Dorothee M./ Moser, Heinz/ Niesyto, Horst (Hg.): Jahrbuch Medienpädagogik 8. Medienkompetenz und Web 2.0. Wiesbaden: VSVerlag, S. 59-79.

Niesyto, Horst/ Holzwarth, Peter/ Maurer, Björn (Hg.) (2007): Interkulturelle Kommunikation mit Foto und Video. München: kopaed.

Schulz-Zander, Renate/ Eickelmann, Birgit (2008): Zur Erfassung von Schulentwicklungsprozessen im Bereich digitaler Medien. In: MedienPädagogik, Zeitschrift für Theorie und Praxis der Medienbildung, Themenheft 14. Online verfügbar unter ht t p : / / www. medien paed.com/14/schulzzander0801.pdf

Tulodziecki, Gerhard (2006): Schulische Medienpädagogik - von den 1950er Jahren bis heute. In: medien + erziehung, 50. Jahrgang, Nr. 5, Oktober 2006, S. 49-56.

Wagner, Ulrike (Hg.) (2008): Medienhandeln in Hauptschulmilieus. München: kopaed.

\section{Zum Autor}

Prof. Dr. Horst Niesyto leitet die Abteilung Medienpädagogik im Institut für Erziehungswissenschaft an der Pädagogischen Hochschule Ludwigsburg. Er ist Vorsitzender der Sektion Medienpädagogik in der Deutschen Gesellschaft für Erziehungswissenschaft (DGfE) und Sprecher der Initiative „Keine Bildung ohne Medien!“" 